Pacific Journal of Mathematics

SOME RESULTS ON COMPLETABILITY IN COMMUTATIVE 


\section{SOME RESULTS ON COMPLETABILITY IN COMMUTATIVE RINGS}

\section{Marion Moore AND Arthur Steger}

In this paper, $R$ always denotes a commutative ring with identity. The ideal of nilpotents and the Jacobson radical of the ring $R$ are denoted by $N(R)$ and $J(R)$, respectively. The vector $\left[a_{1}, \cdots, a_{n}\right]$ is called a primitive row vector provided $1 \in\left(a_{1}, \cdots, a_{n}\right)$; a primitive row vector $\left[a_{1}, \cdots, a_{n}\right]$ is called completable provided there exists an $n \times n$ unimodular matrix over $R$ with first row $a_{1}, \cdots, a_{n}$. A ring $R$ is called a $B$-ring if given a primitive row vector $\left[a_{1}, \cdots, a_{n}\right], n \geqq 3$, and

$$
\left(a_{1}, \cdots, a_{n-2}\right) \nsubseteq J(R),
$$

there exists $b \in R$ such that $1 \in\left(a_{1}, \cdots, a_{n-2}, a_{n-1}+b a_{n}\right)$. Similarly, $R$ is defined to be a Strongly $B$-ring ( $S B$-ring), if $d \in$ $\left(a_{1}, \cdots, a_{n}\right), n \geqq 3$, and $\left(a_{1}, \cdots, a_{n-2}\right) \nsubseteq J(R)$ implies that there exists $b \in R$ such that $d \in\left(a_{1}, \cdots, a_{n-2}, a_{n-1}+b a_{n}\right)$.

in this paper it is proved that every primitive vector over a $B$-ring is completable. It is shown that the following are $B$-rings: $\pi$-regular rings, quasi-semi-local rings, Noetherian rings in which every (proper) prime ideal is maximal, and adequate rings. In addition it is proved that $R[X]$ is a $B$-ring if and only if $R$ is a completely primary ring. It is then shown that the following are $S B$-rings: quasi-local rings, any ring which is both an Hermite ring and a $B$-ring, and Dedekind domains. Finally, it is shown that $R[X]$ is an $S B$-ring if and only if $R$ is a field.

\section{B-rings.}

LEMMA 2.1. Let $R$ be a ring with $A \leqq J(R), A$ an idsal of $R$. Then $R$ is a B-ring if and only if $R / A$ is a $B$-ring.

Proof. Necessity: Let $R$ be a $B$-ring and let

$$
(1+A) \in\left(a_{1}+A, \cdots, a_{n}+A\right), n \geqq 3
$$

and

$$
\left(a_{1}+A, \cdots, a_{n-2}+A\right) \nsubseteq J(R / A)=J(R) / A,
$$

where $a_{i} \in R, i=1, \cdots n$. Then $1+A=\sum_{i=1}^{n} a_{i} b_{i}+A, b_{i} \in R$; hence $\left[a_{1}, \cdots, a_{n}\right]$ is primitive. Since $\left(a_{1}, \cdots, a_{n-2}\right) \nsubseteq J(R)$, it follows that $\left[a_{1}+A, \cdots, a_{n-2}+A,\left(a_{n-1}+b a_{n}\right)+A\right]$ is primitive for some $b \in R$. Therefore, $R / A$ is a $B$-ring.

Sufficiency: Suppose $R / A$ is a $B$-ring and suppose $\left[\alpha_{1}, \cdots, a_{n}\right]$ is a 
primitive vector with $\left(a_{1}, \cdots, a_{n-2}\right) \varsubsetneqq J(R)$. Hence $\left[a_{1}+A, \cdots, a_{n}+A\right]$ is a primitive vector; and, since $\left(a_{1}, \cdots, a_{n-2}\right) \nsubseteq J(R)$, we have $\left(a_{1}+\right.$ $\left.A, \cdots, a_{n-2}+A\right) \nsubseteq J(R / A)$. Since $R / A$ is a $B$-ring, there exists $b+$ $A \in R / A$ such that $\left[a_{1}+A, \cdots, a_{n-2}+A,\left(a_{n-1}+b a_{n}\right)+A\right]$ is primitive. It follows that $(1-u) \in A \subseteq J(R)$, where

$$
u=\sum_{i=1}^{n-2} a_{i} b_{i}+\left(a_{n-1}+b a_{n}\right) b_{n-1}, b_{i} \in R, i=1, \cdots, n-1 .
$$

Therefore, $u$ is a unit of $R$; i.e., $\left[a_{1}, \cdots, a_{n-2}, a_{n-1}+b a_{n}\right]$ is primitive.

THEOREM 2.1. If $R$ is a $B$-ring then every primitive row vector over $R$ is completable.

Proof. Let $R$ be a $B$-ring and let $1 \in\left(a_{1}, \cdots, a_{n}\right)$. The theorem clearly holds for $n=1$. If $n=2$, then $1=a_{1} x+a_{2} y, x, y \in R$ and the matrix $\left(\begin{array}{cc}a_{1} & a_{2} \\ -y & x\end{array}\right)$ is unimodular; hence the result holds for $n=2$.

Let $n \geqq 3$, and suppose the result is established for $k<n$.

Case 1. If $\left(a_{1}, \cdots, a_{n-2}\right) \subseteq J(R)$ and $1=\sum_{i=1}^{n} a_{i} b_{i}, b_{i} \in R$, then $1-$ $\sum_{i=1}^{n-2} a_{i} b_{i}=a_{n-1} b_{n-1}+a_{n} b_{n}$ is a unit $u \in R$. Let

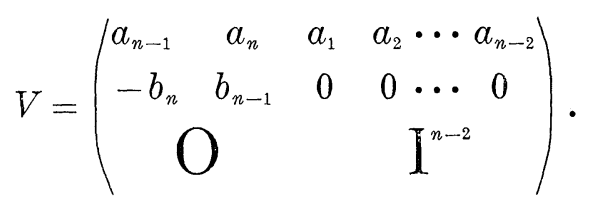

Then $V$ has determinant $u$, and it follows that $\left[a_{1}, \cdots, a_{n}\right]$ is completable.

Case 2. If $\left(a_{1}, \cdots, a_{n-2}\right) \nsubseteq J(R)$, then $1 \in\left(a_{1}, \cdots, a_{n-2}, a_{n-1}+b a_{n}\right)$, for some $b \in R$. By the induction hypothesis, $\left[a_{1}, \cdots, a_{n-2}, a_{n-1}+b a_{n}\right]$ is completable to an $(n-1) \times(n-1)$ unimodular matrix $D$. Let

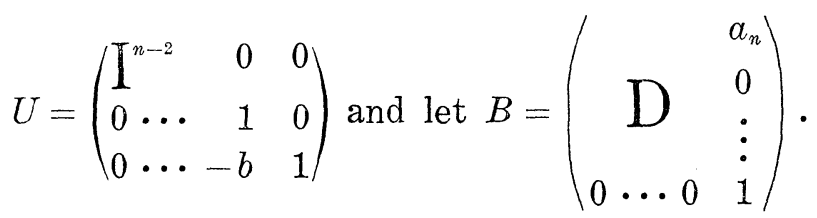

Then $B U$ is an $n \times n$ unimodular matrix whose first row is $\left[a_{1}, \cdots, a_{n}\right]$.

For convenience, we introduce the notation $Z(A)$ to mean the set of maximal ideals containing the ideal $A ; Z(\alpha)$ will denote the set of maximal ideals containing the element $a$.

THEOREM 2.2 If $R$ is a ring such that for every ideal $A \nsubseteq J(R)$, $Z(A)$ is finite, then $R$ is a $B$-ring. 
Proof. The essentials of the proof are due to Reiner [4]. Let $1 \in\left(a_{1}, \cdots, a_{n}\right), n \geqq 3$, and $\left(a_{1}, \cdots, a_{n-2}\right) \nsubseteq J(R)$. By the hypothesis on $R, Z(A)$ is finite where $A=\left(a_{1}, \cdots, a_{n-2}\right)$. Let $Z(A)=\left\{M_{1}, \cdots, M_{r}\right\}$, and note that if $b \in R$ and $a_{n-1}+b a_{n} \in M_{i}, i=1, \cdots, r$, then $\left[a_{1}, \cdots\right.$, $\left.a_{n-2}, a_{n-1}+b a_{n}\right]$ is primitive.

For any $M_{\imath} \in Z(A)$ such that $a_{n} \in M_{i}$, we have $a_{n-1}+b a_{n} \in M_{i}$, for all $b \in R$; otherwise, $a_{n-1} \in M_{i}$, and $\left(a_{1}, \cdots, a_{n}\right) \subseteq M_{i}$ which contradicts the hypothesis that $\left[a_{1}, \cdots, a_{n}\right]$ is primitive.

For those $M_{i} \in Z(A)$ for which $a_{n} \in M_{i}$, we have $\left(a_{n}, M_{i}\right)=(1)$. Hence there exists an $x_{i}$ such that $a_{n} x_{i} \equiv a_{n-1}\left(\bmod M_{i}\right)$. For these $M_{i}$, we can find (by the Chinese Remainder Theorem) an element $b \in R$ such that $b \equiv 1-x_{i}\left(\bmod M_{i}\right)$. It follows that $a_{n-1}+b a_{n} \in M_{i}, i=$ $1, \cdots, r$. Hence $\left[a_{1}, \cdots a_{n-2}, a_{n-1}+b a_{n}\right]$ is primitive.

It follows from this theorem that quasi-semi-local rings and Noetherian rings in which every proper prime ideal is maximal (in particular, Dedekind domains) are $B$-rings.

Lemma 2.2. Let $R$ be an F-ring (i.e., a ring in which every finitely generated ideal is principal) which satisfies the condition that if $1 \in\left(a_{1}, a_{2}, a_{3}\right)$ with $a_{1} \notin J(R)$ thon $1 \in\left(a_{1}, a_{2}+b a_{3}\right)$ for some $b \in R$. Then $R$ is a $B$-ring.

Proof. Let $1 \in\left(a_{1}, \cdots, a_{n}\right), n \geqq 3$, and let $(a)=\left(a_{1}, \cdots, a_{n-2}\right) \varsubsetneqq$ $J(R)$. Hence $1 \in\left(a, a_{n-1}, a_{n}\right)$. By the hypothesis on $R, 1 \in\left(a, a_{n-1}+b a_{n}\right)$; hence, $R$ is a $B$-ring.

THEOREM 2.3. If $R$ is an F-ring which satisfies the condition that for every $a, c \in R$ with $a \in J(R)$, there is an $r \in R$ such that $Z(r)=Z(a)-Z(c)$, then $R$ is a $B$-ring.

Proof. The proof is essentially the same as the proof of Theorem 5 of [2]. Let $1 \in(a, b, c), a \notin J(R)$. By the hypothesis on $R$ there exists $r \in R$ such that $Z(r)=Z(a)-Z(c)$. Hence $(c, r)=(1)$, so there exists $q \in R$ such that $1 \in(r, b+q c)$. We claim $(a, b+q c)=(1)$. Otherwise, there exists a maximal ideal $M$ of $R$ such that $(a, b+q c) \subseteq$ $M$. Hence $M \in Z(a)$ and $M \in Z(b+q c)$. Since $1 \in(r, b+q c)$ it follows that $M \notin Z(r)$, so $M \in Z(c)$. But we now have $M \in Z(b)$, contrary to $(a, b, c)=(1)$. Therefore $(a, b+q c)=(1)$. Lemma 2.2 completes the proof.

Theorem 2.4. Every adequate ring is a B-ring.

Proof. In the proof of Theorem 5.3 of [3], Kaplansky shows that 
if $R$ is an adequate ring and if $1 \in(a, b, c), a \neq 0$, then there exists $q \in R$ such that $1 \in(a, b+q c)$. Since an adequate ring is an $F$-ring, the result follows from Lemma 2.2 .

\section{THEOREM 2.5. Every $\pi$-regular ring is a B-ring.}

Proof. If $R$ is a $\pi$-regular ring, and if $a \in R / N(R)$, then by Lemma 2.2 of [5], $a$ is an associate of $e+\beta, e$ an idempotent and $\beta$ a nilpotent of the $\pi$-regular ring $R / N(R)$. Since $\beta=0, a=u e, u$ a unit of $R / N(R)$. Therefore, $a^{2}=u^{2} e$ and $u^{-1} a^{2}=u e=a$. Hence, $R / N(R)$ is a regular ring and therefore an adequate ring ([1, Th. 11]). Theorem 2.4 and Lemma 2.1 complete the proof.

THEOREM 2.6. Let $D$ be an integral domain, $K$ its quotient field. Let $R=\left\{\left(a_{1}, \cdots, a_{k}, a, a, \cdots\right): a_{i} \in K, a \in D\right\}$, where $k$ is a nonnegative integer ( $k$ may be different for distinct elements of $R$ ). The operations in $R$ are component-wise addition and multiplication. If $R$ is a $B$ ring then $D$ is a B-domain.

We illustrate the proof. Suppose $R$ is a $B$-ring and let $1 \in(a, b, c)$, $a, b, c \in D, 1=a a^{\prime}+b b^{\prime}+c c^{\prime}$. Let $\hat{a}=(1, a, a, \cdots), \hat{b}=(0, b, b, \cdots)$, $\left.\hat{c}=(0, c, \cdots), \hat{a}^{\prime}=\left(1, a^{\prime}, a^{\prime}, \cdots\right), \hat{b}^{\prime}=\left(0, b^{\prime}, b^{\prime}, \cdots\right), \hat{c}^{\prime}=0, c^{\prime}, c^{\prime}, \cdots\right)$. Then $\hat{1}=\hat{a} \hat{a}^{\prime}+\hat{b} \hat{b}^{\prime}+\hat{c} \hat{c}^{\prime}$. If $\hat{a} \in J(R)$, then $\hat{1}-\hat{a}=(0,1-a, 1-a, \cdots)$ is a unit of $R$. Since this is false, $\hat{a} \notin J(R)$, hence $\hat{1} \in(\hat{a}, \hat{b}+\hat{y} \hat{c})$ for some $\hat{y} \in R$. Therefore $\hat{1}=\hat{a} \hat{d}+(\hat{b}+\hat{y} \hat{c}) \hat{e}$, where $\hat{d}, \hat{c}, \hat{e} \in R$. Let $\hat{d}=$ $\left(d_{1}, \cdots, d_{p}, d, d, \cdots\right), \hat{e}=\left(e_{1}, \cdots, e_{q}, e, e, \cdots\right), \hat{y}=\left(y_{1}, \cdots, y_{r}, y, y, \cdots\right)$ and let $\lambda=\max (1, p, q, r)$. In the $(\lambda+1)$ st entry of $\hat{a} \hat{d}+(\hat{b}+\hat{y} \hat{c}) \hat{e}$, we have $a d+(b+y c) e$; i.e., $1 \in(a, b+y c)$. Hence, $D$ is a $B$-domain.

THEOREM 2.7. $R[X]$ is a B-ring if and only if $R$ is a completely primary ring.

Proof. Sufficiency: Let $R$ be a completely primary ring. Since $R / N(R)$ is a field and since $(R / N(R))[X] \cong R[X] / N(R)[X]$, it follows from Theorem 2.2 that $R[X] / N(R)[X]$ is a $B$-ring. Since $N(R)[X]=$ $N(R[X])$, the result follows from Lemma 1.2.1.

Necessity: Assume that $R$ is not completely primary and that $R[X]$ is a $B$-ring. Let $r$ be a nonunit, nonnilpotent element of $R$. Then $1 \in\left(r, 1+X, X^{2}\right)$ and $r \notin J(R[X])$. By the assumption that $R[X]$ is a $B$-ring, we have $1 \in\left(r, 1+X+X^{2} f(X)\right)$ for some $f(X) \in R[X]$. Let $\bar{a}$ denote the image of $a \in R$ under the natural homomorphism of $R[X]$ onto $(R / r R)[X]$. Then $\overline{1} \in\left(\overline{0}, \overline{1}+X+X^{2} \bar{f}(X)\right)$ and $\overline{1}+X+$ $X^{2} \bar{f}(X)$ is a unit of $(R / r R)[X]$. This is a contradiction since the coefficient of $X$ is not nilpotent. 
Since $R[X]$ cannot be completely primary, (clearly, $X$ is neither a unit nor a nilpotent) it follows that for every ring $R, R[X, Y]=$ $R[X][Y]$ is not a $B$-ring.

3. Strongly $B$-rings. We now turn our attention to the study of $S B$-rings. Our main objective here is to compare the theory of this particular subclass of $B$-rings with that of $B$-rings given in the last section.

LEMma 3.1. $R$ is an $S B$-ring if and only if for every $s, c_{1}, c_{2}$, $c_{3} \in R$ with $s \in\left(c_{1}, c_{2}, c_{3}\right)$ and $c_{1} \in J(R)$, it follows that $s \in\left(c_{1}, c_{2}+b c_{3}\right)$ for some $b \in R$.

Proof. The necessity clearly follows from the definition of an $S B$ ring.

Sufficiency: Let $r \in\left(a_{1}, \cdots, a_{n}\right), n \geqq 3$, with $\left(a_{1}, \cdots, a_{n-2}\right) \nsubseteq J(R)$. Without loss of generality, we may assume that $a_{n-2} \in J(R)$. Suppose $r=\sum_{i=1}^{n} a_{i} x_{i}$ and let $s=a_{n-2} x_{n-2}+a_{n-1} x_{n-1}+a_{n} x_{n}$. Then $r \in\left(a_{1}, \cdots\right.$, $\left.a_{n-3}, s\right)$ and $s \in\left(a_{n-2}, a_{n-1}, a_{n}\right)$. Since $a_{n-2} \in J(R), s \in\left(a_{n-2}, a_{n-1}+b a_{n}\right)$ for some $b \in R$. Therefore $r \in\left(a_{1}, \cdots, a_{n-3}, s\right) \leqq\left(a_{1}, \cdots, a_{n-1}+b a_{n}\right)$, and the proof is complete.

In view of Lemma 3.1, we need only consider triples instead of arbitrary $n$-tuples in our study of $S B$-rings.

LEMma 3.2. The homomorphic image of an SB-ring is an $S B$ ring.

Proof. Let $\bar{R}$ be the image of $R$ under the homomorphism $\dot{\phi}$, and let $\bar{d} \in\left(\bar{a}_{1}, \bar{a}_{2}, \bar{a}_{3}\right)$ with $\bar{a}_{1} \in J(\bar{R}), \bar{a}_{1}, \bar{a}_{2}, \bar{a}_{3}, \bar{d} \in \bar{R}$. Suppose $\bar{d}=\sum_{i=1}^{3} \bar{a}_{i} \bar{x}_{i}$, $\bar{x}_{i} \in \bar{R}$ and let $a_{i} \dot{\phi}=\bar{a}_{i}, x_{i} \dot{\phi}=\bar{x}_{i}, i=1,2,3$. Let $d=\sum_{i=1}^{s} a_{i} x_{i}$. Since $(J(R)) \phi \leqq J(\bar{R})$, we have $a_{1} \in J(R)$; hence, $d \in\left(a_{1}, a_{2}+b a_{3}\right)$ for some $b \in R$. Since $d \dot{\phi}=\bar{d}$, we have $\bar{d} \in\left(\bar{a}_{1}, \bar{a}_{2}+\bar{b} \bar{a}_{3}\right)$, where $b \dot{\phi}=\bar{b}$. Hence $\bar{R}$ is an $S B$-ring.

THEOREM 3.1. Every quasi-local ring is an SB-ring.

Proof. Let $d \in\left(a_{1}, a_{2}, a_{3}\right)$, with $a_{1} \in J(R), R$ a quasi-local ring. Since $a_{1} \in J(R), a_{1}$ is a unit of $R$; hence, $\mathrm{d} \in\left(a_{1}, a_{2}+b a_{3}\right)=(1)$ for every $b \in R$.

Lemma 3.3. Let $A=\left(a_{1}, \cdots, a_{n}\right), n \geqq 3$, be an ideal in a Dedekind domain $R$. If $B=\left(a_{1}, \cdots, a_{n-2}\right) \neq(0)$, then $A=\left(a_{1}, \cdots, a_{n-2}, a_{n-1}+\right.$ $\left.b a_{n}\right)$ for some $b \in R$. 
Proof. Let $A=\prod_{i=1}^{t} M_{i}^{\alpha_{i}}$ and let $B=\prod_{i=1}^{t} M_{i}^{\beta}$ be the representations of the ideals $A$ and $B$ as a product of powers of distinct maximal ideals. Since $B \leqq A$, we may order the $M_{i}$ so that $0 \leqq \alpha_{i}<\beta_{i}$ for $1 \leqq i \leqq r$, and $\alpha_{i}=\beta_{i}$ for $r+1 \leqq i \leqq t$. Let $1 \leqq k \leqq r$. We claim that either $a_{n-1}$ or $a_{n}$ does not belong to $M_{k}^{\alpha} k^{+1}$. For suppose both $a_{n-1}$ and $a_{n}$ belong to $M_{k}^{\alpha} k^{+1}$. Then $A \subseteq M_{k}^{\alpha} k_{k}^{+1}$, a contradiction. Since the $M_{k}^{\alpha} \alpha^{\alpha} \div 1$ are relative prime, the Chinese Remainder Theorem guarantees the existence of a $b \in R$ satisfying:

$$
\begin{aligned}
& b \equiv 0\left(\bmod M_{k}^{\alpha_{k}+1}\right) \quad \text { if } \quad a_{n-1} \in M_{k}^{\alpha_{k}+1} \\
& b \equiv 1\left(\bmod M_{k}^{\alpha_{k}+1}\right) \text { if } a_{n-1} \in M_{k}^{\alpha_{k}+1} \text {, }
\end{aligned}
$$

for $k=1,2, \cdots, r$. It follows that $a_{n-1}+b a_{n} \notin M_{k}^{\alpha} k^{+1}$ for $k=1,2, \cdots, r$. Let $\left(a_{1}, \cdots, a_{n-2}, a_{n-1}+b a_{n}\right)=\prod_{i=1}^{t} M_{i}^{\prime \prime}$. Since $\left(a_{1}, \cdots, a_{n-2}, a_{n-1}+b a_{n}\right) \subseteq$ $A=\prod_{i=1}^{t} M_{i}^{\kappa_{i}}$, it follows that $\mu_{i} \geqq \alpha_{i}, i=1,2, \cdots, t$. Since $B=\prod_{i=1}^{t}$ $M_{i}^{\beta i} \subseteq \prod_{i=1}^{t} M_{i}^{\prime \prime} \subseteq \subseteq \prod_{i=1}^{t} M_{i}^{\gamma_{i}}=A$, and since $\beta_{i}=\alpha_{i}, r+1 \leqq i \leqq t$, it follows that $\mu_{i}=\beta_{i}=\alpha_{i}, r+1 \leqq i \leqq t$. If $\mu_{i}>\alpha_{i}$ for some $i$ with $1 \leqq i \leqq r$, then $a_{n-1}+b a_{n} \in M_{i}^{\mu_{i}} \subseteq M_{i}^{\alpha_{i+1}}$, a contradiction. Hence, $\mu_{i}=$ $\alpha_{i}, i=1,2, \cdots, t$. Equivalently, $\left(a_{1}, \cdots, a_{n-2}, a_{n-1}+b a_{n}\right)=A$.

As an immediate consequence, we have:

TheOREM 3.2. A Dedekind domain is an SB-ring.

Lemma 3.4. Let $R$ be a $B$ - $r$ ing, let $e=e^{2} \in R$, and let $e \in\left(a_{1}, \cdots, a_{n}\right)$ with $\left(a_{1}, \cdots, a_{n-2}\right) \nsubseteq J(R), n \geqq 3$. Then $e \in\left(a_{1}, \cdots, a_{n-2}, a_{n-1}+b a_{n}\right)$ for some $b \in R$.

Proof. Since the case $e=1$ is covered by the hypothesis, we may assume $e \neq 1$. Let $e=\sum_{i=1}^{n} a_{i} x_{i}=\sum_{i=1}^{n}\left(a_{i} e\right)\left(x_{i} e\right)$. Hence, $1=$ $\left(a_{1} e+1-e\right)\left(x_{1} e+1-e\right)+\sum_{i=2}^{n}\left(a_{i} e\right)\left(x_{i} e\right)$. Thus,

$$
1 \in\left(a_{1} e+1-e, a_{2} e, \cdots, a_{n} e\right) \text {. }
$$

If $a_{1} e+1-e \in J(R)$, then $1-\left(a_{1} e+1-e\right)=e\left(1-a_{1}\right)$ is a unit of $R$, a contradiction since $e=e^{2}, e \neq 1$. Thus, since $R$ is a $B$-ring, we have $1 \in\left(a_{1} e+1-e, a_{2} e, \cdots, a_{n-2} e, a_{n-1} e+b a_{n} e\right)$ for some $b \in R$. Therefore, $e \in\left(a_{1} e, a_{2} e, \cdots, a_{n-2} e, a_{n-1} e+b a_{n} e\right) \leqq\left(a_{1}, a_{2}, \cdots, a_{n-2}, a_{n-1}+b a_{n}\right)$.

COROLlaRy. If $R$ is a regular ring then $R$ is an $S B$-ring.

Proof. The result is immediate from Theorem 2.5 and Lemma 3.4; since, for every $r \in R, r$ is an associate of some idempotent $e \in R$ ([1, Lemma 10]).

THEOREM 3.3. If a B-ring $R$ is also an Hermite ring, then $R$ is an $S B$-ring. 
Proof. Let $d \in\left(a_{1}, a_{2}, a_{3}\right)=(a), a_{1} \in J(R)$. By Corollary 5 of [1], there exist $b_{1}, b_{2}, b_{3}$ such that $a_{1}=b_{1} a, a_{2}=b_{2} a, a_{3}=b_{3} a$, and $\left(b_{1}, b_{2}, b_{3}\right)=$ (1). Since $R$ is a $B$-ring and since $b_{1} \in J(R)$, there exists a $q \in R$ such that $\left(b_{1}, b_{2}+q b_{3}\right)=(1)$. Therefore, $(a)=\left(b_{1} a, b_{2} a+q b_{3} a\right)=\left(a_{1}, a_{2}+q a_{3}\right)$. Hence, $d \in\left(a_{1}, a_{2}+q a_{3}\right)$.

COROLlary. Every adequate domain is an SB-ring.

Proof. An adequate domain is both an $F$-domain and a $B$-ring. Since every $F$-domain is an Hermite ring, the result follows from Theorem 3.3.

CoROllary. If $R$ is an F-ring with infinitely many maximal ideals and, if for every ideal $A \nsubseteq J(R), Z(A)$ is finite, then $R$ is an SB-ring.

Proof. $R$ is necessarily a $B$-ring by Theorem 2.2. By the proof of Corollary 2 of [2], $R$ is also an Hermite ring. Theorem $3.3 \mathrm{com}$ pletes the proof.

Theorem 3.4. $R[X]$ is an SB-ring if and on'y if $R$ is a field.

Proof. The sufficiency follows from Theorem 3.2. To prove the necessity, let $r \in R, r \neq 0$. Then $r \in\left(X^{2}, X, r\right)$ and $X^{2} \in J(R[X])$. If $R[X]$ is an $S B$-ring then $r \in\left(X^{2}, X+r b(X)\right)$ for some $b(X) \in R[X]$. Let $r=X^{2} f(X)+(X+r b(X)) g(X)$, where $f(X)$ and $g(X) \in R[X]$, and let $f_{i}, g_{i}, b_{i}$ represent the coefficient of $X^{i}$ in the polynomials $f(X)$, $g(X), b(X)$, respectively. Equating coefficients in the above equation gives $r=r_{0} b_{0} g_{0}$ and $0=g_{0}+r\left(b_{0} g_{1}+g_{0} b_{1}\right)$. Hence $r$ divides $g_{0}$ and therefore $r=r^{2} k$ for some $k \in R$. Hence $r k=(r k)^{2}$; therefore, $r k$ is an idempotent of $R$. Since $R[X]$ is a $B$-ring, $R$ must be a completely primary ring by Theorem 2.7. It follows that the idempotent $r k$ is either 0 or 1 . Since $r k=0$ and $r=r^{2} k$ imply $r=0$, we conclude that $r k=1$; i.e., $r$ is a unit of $R$. Hence, $R$ is a field and the proof is complete.

\section{REFERENCES}

1. L. Gillman and M. Henriksen, Some remarks about elementary divisor rings, Trans. Amer. Math. Soc. 82 (1956), 362-365.

2. M. Henriksen, Some remarks on elementary divisor rings II, Michigan Math. J. 3 (1955-56), 159-163.

3. I. Kaplansky, Elementary divisors and modules, Trans. Amer. Math. Soc. 66 (1959), 464-491.

4. I. Reiner, Unimodular complements, Amer. Math. Monthly 63 (1956), 246-247. 
5. A. Steger, Elementary factorization in $\pi$-regular rings, Canad. J. Math. 18 (1966), 307-313.

Received October 30, 1969.

University of Texas at Arlington

University of New Mexico 


\section{PACIFIC JOURNAL OF MATHEMATICS}

\section{EDITORS}

\author{
H. SAMElson \\ Stanford University \\ Stanford, California 94305 \\ C. R. HOBBY \\ University of Washington \\ Seattle, Washington 98105
}

J. DugundjI

Department of Mathematics University of Southern California Los Angeles, California 90007

RICHARD ARENS

University of California

Los Angeles, California 90024

\section{ASSOCIATE EDITORS}
E. F. BECKENBACH
B. H. NeUmanN
F. WOLE
K. YOSHIDA

\section{SUPPORTING INSTITUTIONS}

\author{
UNIVERSITY OF BRITISH COLUMBIA \\ CALIFORNIA INSTITUTE OF TECHNOLOGY \\ UNIVERSITY OF CALIFORNIA \\ MONTANA STATE UNIVERSITY \\ UNIVERSITY OF NEVADA \\ NEW MEXICO STATE UNIVERSITY \\ OREGON STATE UNIVERSITY \\ UNIVERSITY OF OREGON \\ OSAKA UNIVERSITY \\ UNIVERSITY OF SOUTHERN CALIFORNIA
}

\author{
STANFORD UNIVERSITY \\ UNIVERSITY OF TOKYO \\ UNIVERSITY OF UTAH \\ WASHINGTON STATE UNIVERSITY \\ UNIVERSITY OF WASHINGTON \\ * * * \\ AMERICAN MATHEMATICAL SOCIETY \\ CHEVRON RESEARCH CORPORATION \\ NAVAL WEAPONS CENTER
}

The Supporting Institutions listed above contribute to the cost of publication of this Journal, but they are not owners or publishers and have no responsibility for its content or policies.

Mathematical papers intended for publication in the Pacific Journal of Mathematics should be in typed form or offset-reproduced, (not dittoed), double spaced with large margins. Underline Greek letters in red, German in green, and script in blue. The first paragraph or two must be capable of being used separately as a synopsis of the entire paper. The editorial "we" must not be used in the synopsis, and items of the bibliography should not be cited there unless absolutely necessary, in which case they must be identified by author and Journal, rather than by item number. Manuscripts, in duplicate if possible, may be sent to any one of the four editors. Please classify according to the scheme of Math. Rev. Index to Vol. 39. All other communications to the editors should be addressed to the managing editor, Richard Arens, University of California, Los Angeles, California, 90024.

50 reprints are provided free for each article; additional copies may be obtained at cost in multiples of 50 .

The Pacific Journal of Mathematics is published monthly. Effective with Volume 16 the price per volume (3 numbers) is $\$ 8.00$; single issues, $\$ 3.00$. Special price for current issues to individual faculty members of supporting institutions and to individual members of the American Mathematical Society: $\$ 4.00$ per volume; single issues $\$ 1.50$. Back numbers are available.

Subscriptions, orders for back numbers, and changes of address should be sent to Pacific Journal of Mathematics, 103 Highland Boulevard, Berkeley, California, 94708.

PUBLISHED BY PACIFIC JOURNAL OF MATHEMATICS, A NON-PROFIT CORPORATION

Printed at Kokusai Bunken Insatsusha (International Academic Printing Co., Ltd.), 7-17, Fujimi 2-chome, Chiyoda-ku, Tokyo, Japan. 


\section{Pacific Journal of Mathematics}

\section{Vol. 37, No. $2 \quad$ February, 1971}

Charles Compton Alexander, Semi-developable spaces and quotient images of metric spaces .................................... 277

Ram Prakash Bambah and Alan C. Woods, On a problem of Danzer. . . . . . . . . 295

John A. Beekman and Ralph A. Kallman, Gaussian Markov expectations and related integral equations . ....................................

Frank Michael Cholewinski and Deborah Tepper Haimo, Inversion of the Hankel

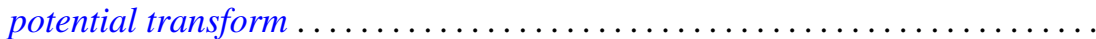

John H. E. Cohn, The diophantine equation

$$
Y(Y+1)(Y+2)(Y+3)=2 X(X+1)(X+2)(X+3) \ldots \ldots \ldots \ldots \ldots
$$

Philip C. Curtis, Jr. and Henrik Stetkaer, A factorization theorem for analytic

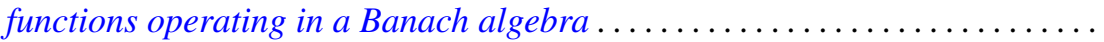

Doyle Otis Cutler and Paul F. Dubois, Generalized final rank for arbitrary limit ordinals

Keith A. Ekblaw, The functions of bounded index as a subspace of a space of entire functions

Dennis Michael Girard, The asymptotic behavior of norms of powers of

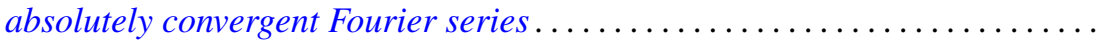

John Gregory, An approximation theory for elliptic quadratic forms on Hilbert spaces: Application to the eigenvalue problem for compact quadratic forms. 383

Paul C. Kainen, Universal coefficient theorems for generalized homology and stable cohomotopy.

Aldo Joram Lazar and James Ronald Retherford, Nuclear spaces, Schauder bases, and Choquet simplexes.

David Lowell Lovelady, Algebraic structure for a set of nonlinear integral operations

John McDonald, Compact convex sets with the equal support property . 429

Forrest Miller, Quasivector topologies

Marion Edward Moore and Arthur Steger, Some results on completability in commutative rings.

A. P. Morse, Taylor's theorem

Richard E. Phillips, Derek J. S. Robinson and James Edward Roseblade, Maximal subgroups and chief factors of certain generalized soluble groups.

Doron Ravdin, On extensions of homeomorphisms to homeomorphisms ...

John William Rosenthal, Relations not determining the structure of $\mathrm{L}$

Prem Lal Sharma, Proximity bases and subbases ........... .

Larry Smith, On ideals in $\Omega_{*}^{u}$. .

Warren R. Wogen, von Neumann algebras generated by operators similar to normal operators

R. Grant Woods, Co-absolutes of remainders of Stone-Čech 